\title{
The resources of an inclusive educational environment in the development of thinking of primary students with psychical developmental delay
}

\author{
Olga Solovieva*, Natalia Borozinets, Nadezhda Palieva, and Galina Kozlovskaya \\ North Caucasus Federal University, Stavropol, Russia
}

\begin{abstract}
This article describes the problem of studying the resources of an inclusive educational environment in the development of thinking of primary students with psychical developmental delay. A brief analytical review of the degree of development of this problem is presented. The characteristic of the specific development of the mental activity of primary students with psychical developmental delay is disclosed. A program is proposed for the purposeful development of thinking of children with psychical developmental delay; the psychological and pedagogical conditions for the effectiveness of this process in an inclusive educational environment are identified.
\end{abstract}

The thinking is the highest stage of human cognition. This is a process of generalized and indirect reflection of stable regular properties and relationships of reality for solving cognitive problems. Thinking is characterized by an active search for connections and relationships between events, phenomena, things, objects.

In primary school age, thinking is the center of the child's mental development and determines the work of other thinking processes that become arbitrary and conscious. Therefore, thinking at this age must be develop. An important issue of educational psychology is the search for means, methods and conditions for the development of thinking in children.

Of particular importance is the focused development and correction of thinking in children with psychical developmental delay. This is due to the fact that they are characterized by underdevelopment of the cognitive sphere, a poor supply of information about the world around them, a low level of cognitive activity, deficiencies in the development of speech, difficulties in solving educational and practical tasks, difficulties in performing such mental operations as generalization, classification, systematization and concretization.

All this indicates a lack of development of thinking in this category of children, so they have difficulty learning. The success of further education of the child, and its social adaptation, largely depend on the development of thinking.

* Corresponding author: olga.vl.soloveva@gmail.com 
Children with psychical developmental delay constitute the largest group included general educational practice. Therefore, the urgent task of their training is to determine the resources of an inclusive educational environment and to identify the psychological and pedagogical conditions for the development of thinking in an inclusive educational process. The problem of the development of children thinking has been research by many scientists. Theoretical aspects are presented in the works of A. Brushlinsky, L. Vygotsky, V. Davydov, S. Rubinstein, A. Luria, A. Leontiev, N.N. Pospelov, J. Piaget, L. Tikhomirov, M. Kholodnaya, D. Elkonin et al.

Various approaches to the development of thinking of primary students are presented in the works of P. Blonsky, L. Vygotsky, D. Elkonin, Z. Zak, N. Menchinskaya, A. Matyushkin, A. Meshcheryakova and others. The theory of the gradual formation of mental actions of primary school children is presented in the works of P. Galperin, A. Zaporozhets, G. Kostyuk.

Peculiarities of the psyche and thinking of children with psychical developmental delay are reflected in works of T. Vlasova, G. Zharenkova, T. Egorova, V. Lebeninsky, K. Lebedinskaya V. Lubovsky, M. Pevzner, T. Puskayeva, G. Sukhareva, S. Shevchenko, U. Ulenkova et al.

A theoretical analysis of special psychological literature indicates that the extreme complexity and multifaceted nature of the problem of the development of thinking puts forward certain requirements for identifying its features and determining the conditions for targeted development in primary students with psychical developmental delay.

An analysis of practical experience shows that many primary students with psychical developmental delay have a low level of development of verbal-logical thinking, an inability to establish connections and relationships between objects and phenomena, not criticality in assessing their cognitive activity. At the same time, educational psychologists of educational institutions do not know the methods and means of focused work on the development of thinking, its operations and functionality, taking into account the peculiarities of the delay in the development of thinking processes. Therefore, there are contradictions between:

- the need to develop thinking of primary students with psychical developmental delay and the absence of a system for its targeted development in the process of psychological support in educational organizations;

- the need to improve the mental and creative abilities of children with psychical developmental delay in the process of introducing them to the world of information culture and the lack of conditions in an inclusive educational organizations for the full realization of this task.

Based on the contradictions, the research problem consists in finding ways to purposefully develop the thinking of primary students with psychical developmental delay and in substantiating the psychological and pedagogical conditions of this process in an inclusive educational environment.

The purpose of the study is to identify methods, means and psychological and pedagogical conditions for the development of thinking in primary students with psychical developmental delay in an inclusive educational process.

Research objectives: to analyze the resources of an inclusive educational environment for the development of thinking of primary students with psychical developmental delay, to create a program for the development of mental activity primary students of this category and justify the psychological and pedagogical conditions of this process.

The theoretical and methodological basis of the study was theories of thinking (S. Rubinshtein, L. Vygotsky, J. Piaget), studies on the problems of the development of children's thinking (P. Blonsky, D. Bogoyavlensky, A. Brushlinsky, L. Vekker, L. Vygotsky, K. Lebedinskaya, A. Leontiev, N. Menchinskaya, etc.), studies of the mental 
abilities of children with psychical developmental delay (T. Vlasova, G. Zharenkova, K. Lebedinskaya, V. Lubovsky, M. Pevzner, G. Sukharev, S. Shevchenko, U. Ulenkova, etc.), conceptual provisions of educational psychology (A. Boyko, A. Zak, O. Solovyova, S. Yakovleva, etc.), the concepts of activity-oriented and personality-oriented approaches to teaching children (V. Bespalko, V. Davydov, G. Zhelezovskaya, P. Pidkasisty, V. Serikov, I. Yakimanskaya, etc.), learning theories of P. Galperin, L. Zankov, V. Davydov.

Research methods: overview-analytical method - theoretical analysis of the literature on the research problem and empirical methods - testing, observation, conversation, methods of statistical data processing.

An analytical review of the developmental features of primary students with psychical developmental delay showed that in the structure of their condition the main ones are the difficulties of arbitrary regulation of behavior and activity, as well as signs of general socio-emotional immaturity.

In the research of T. Vlasova, T. Egorova, I. Kulagina, V. Lubovsky, M. Pevzner, T. Puskayeva, T. Strekalova, U. Ulenkova, S. Shevchenko, N. Tsipina et al. Noted that for children with psychical developmental delay, a violation of cognitive activity is characteristic, namely a limited supply of knowledge and ideas about the world. They have insufficiently developed attention, memory and thinking, they quickly become tired during intellectual studies. Often there is a slower pace of development of mental processes.

Scientists also note a decreased activity of cognitive activity, insufficient formation of mental operations in children with psychical developmental delay in the learning process at school. Such students do not distinguish the attributes of objects well enough, find it difficult to mentally combine or perform an analysis of the properties of objects and phenomena, carry out orientation in space, and use the stock of knowledge and ideas in practical activities. T. Vlasova, V. Lubovsky, N. Tsypina notes that students with psychical developmental delay experience difficulties in creating a whole from the parts and isolating parts from the whole. For them, spatial manipulation of images seems difficult. They are slower than ordinary children to solve visual-figurative problems and are especially difficult to solve verbal problems [1].

T.V. Egorova draws attention to the reproductive nature of the activities of children with psychical developmental delay. They mainly mechanical reproduce of a small volume material. The process of forming mental operations (analysis, synthesis, comparison, classification, generalization) has been slow [2].

The main drawback of the mental activity of children with psychical developmental delay is the reduced possibility of generalization. V. Lebedinsky notes that primary students can produce mainly only the simplest situational generalizations, as well as generalizations into categories based on the generic names they have learned. They cannot carry out generalizations on unfamiliar material [3].

In the research of B. Puzanov also noted that in children with psychical developmental delay, the scope of comparison of objects is narrowed, differentiation of objects having similar signs and properties is difficult. Unfamiliar objects are generalized according to single signs [4].

Partial difficulties in mastering subject knowledge are typical for children with psychical developmental delay. This is due to impaired mental performance, low productivity during class, the slowed pace of work and a long concentration of attention on the implementation of intellectual cognitive tasks [1-4].

However, in some cases, the general level of development of thinking of children with psychical developmental delay approaches the age norm [3]. Children can be included in the educational process and satisfactorily master the prymary curriculum. It is important to note here that a sufficient level of learning for children with psychical developmental delay is possible with stimulating and organizing assistance. It is timely corrective and 
developmental work, adequate training and education that can compensate for the lack of thinking in children with psychical developmental delay, having formed the necessary stock of knowledge and skills and ways of mental actions.

The conditions of an inclusive educational environment are acceptable and favorable for the development of children with psychical developmental delay. An inclusive approach is an innovative model of the Russian education system at the present stage. The greatest contribution to the development of the conceptual foundations of domestic inclusive education was made by S. Alekhina, E. Ekzhanova, R. Dimenstein, S. Kudinov, E. Kutepova, I. Levchenko, I. Larikova, N. Malofeev, A. Naumov, N. Shmatko, E. Yarskaya-Smirnova et al. Scientists study the subject of inclusive education, develop technologies for inclusive education and inclusive educational practices.

Inclusion implies access to education for all children. The main task is to adapt an educational practices to the needs of each child, regardless of their state of health. Inclusive education provides an opportunity for children with psychical developmental delay to gain social experience in the process of communicating with peers on an equal footing. It is assumed that a child with a psychical developmental delay will receive an education comparable in final level with the formation of normally developing peers.

Mandatory in this case is the creation of adequate conditions to meet the special educational needs of children with psychical developmental delay [5].

An experimental study was conducted on the basis of the inclusive school number 21 in Stavropol. The participants in the experiment were 8 second-grade students with psychical developmental delay. To identify the level of development of their thinking, we used the method of E. Zambacevichene, consisting of four subtests: "Finish the sentence", "Classification - the ability to generalize", "Inference by analogy", "Generalization" [6].

The results of testing, observation of children in the classroom, and conversation with the teacher showed that the characteristic features of thinking of children with psychical developmental delay are a reduced level of generalization, poor classification ability, lack of analytical skills, and great difficulty in completing inferences by analogy. In their judgments, the predominance of spontaneous representations of objects and phenomena, the establishment of specific relationships between objects. Thus, the main features of the thinking of children with psychical developmental delay identified at the stage of the ascertaining experiment is that they have slowed down the process of forming mental operations (analysis, synthesis, comparison, classification, generalization). This is due to the low level of cognitive activity, a small supply of knowledge and low awareness of children with mental retardation, which confirms the theoretical conclusions.

Based on the data of a experiment, we have developed a program for the purposeful development of students' thinking with a psychical developmental delay as one of the most important factors in the success of their learning and general socialization in an inclusive educational environment in primary school.

Given the variability of students' mental activity with psychical developmental delay, correctional and developmental work is most effective provided that an individual approach to each primary student is implemented. In an inclusive class, where children with normal development and children with psychical developmental delay are taught, it is not always possible to devote enough attention and time to an individual approach. It is most convenient to implement in extracurricular activities through special classes. The most acceptable form of such classes, in our opinion, is intellectual training.

E. Zaika notes that the training sessions are useful to all primary students and especially those who experience marked difficulties in performing various types of educational work: understanding and comprehending new material, memorizing and assimilating it, establishing connections between various phenomena, solving theoretical and practical problems, expressing thoughts in speech [7]. 
The training we created is aimed at improving the structure of all cognitive activity of primary students includin mental operations. The content of the training includes such games, tasks and exercises that contribute to the development of verbal-logical, abstract, abstract thinking, as well as its qualities such as depth, flexibility, and criticality. We collected material for training on the development of thinking from various psychological and pedagogical sources (E. Zaika [7], A. Zak [8], O. Solovieva [9], G. Uruntaeva [10] and etc.). Some of the tasks were modified and adapted to the conditions of our formative experiment.

In the course of the training, favorable conditions for spontaneity and high cognitive motivation were created and maintained, which contributed to greater productivity of intellectual activity. Children were happy to attend these classes, because the training provided for a situation of emotional involvement, ease, the possibility of direct communication with peers. Joint activities, a climate of trust and dialogue in the group are a positive factor in interpersonal perception. The training creates favorable conditions for creating a positive educational and cognitive motivation and the implementation of an individual approach. The use of game, entertaining and unusual tasks helps to increase the creative activity of primary students a more meaningful and faster development of individual techniques and means of development of thinking.

When developing the thinking of primary students with a psychical developmental delay, we propose to be guided by the following principles:

- the principle of continuity, aimed at the gradual introduction of material of increasing complexity and the repetition of the passed;

- the principle of humanistic psychology in the relationships of group members, which provides for maintaining a climate of mutual trust, acceptance of others, and psychological safety.

Reliance on these principles in creating motivation and the rational use of developing methods and techniques helps to improve the thinking of a primary students with psychical developmental delay. This compensates for information and mental overload, provides more productive educational activities and better adaptation and socialization of the child in an inclusive educational environment.

It is important to note that training sessions with children with psychical developmental delay in an inclusive class are held together with children of normal development. This is a good incentive in order to focus on higher indicators of mental activity and a variety of ways to solve mental tasks.

The program of the formative experiment in addition to the implementation of the training included work with parents. Active support and parental assistance is required to regularly perform specially selected exercises at home. The regularity of classes contributes to the effectiveness of the development of mental activity as complex systems of conditional connections in the cerebral cortex. We emphasize that simple exercises with regular use and gradual complication contribute to the development of the ability to verbalize the thought process, carry out thought operations and solve mental problems. The psychological and pedagogical conditions for the implementation of the formative program were as follows:

- $\quad$ systematic use of special games and exercises with consistently complicated content in the process of intellectual training;

- the formation of perseverance, determination and other volitional qualities in younger students with a psychical developmental delay in the process of solving mental tasks and exercises;

- $\quad$ an individual approach used to ensure cognitive interest and activity of the child during intellectual training and in lessons;

- $\quad$ work with parents aimed at organizing joint activities to develop the thinking of children 
with psychical developmental delay after school hours;

- organization of interpersonal interaction and communication skills in a group of peers and with adults, which are factors in the personal and cognitive development of a primary student with a psychical developmental delay;

- the use of resources of an inclusive educational environment during class and extracurricular activities, involving the active inclusion of a child with a psychical developmental delay in school life.

We emphasize that children with psychical developmental delay has gained a cognitive interest, the stability of which determines a positive and active attitude towards learning at school, which is the basis for the full assimilation of knowledge, skills.

Thus, from an analysis of the experimental data presented, it can be concluded that under the influence of special developmental classes conducted in the form of training, adherence to principles and the provision of appropriate psychological and pedagogical conditions for primary students with psychical developmental delay, the level of development of thinking has increased and the characteristics of mental activity have changed. In conclusion, we note that the resources of an inclusive educational environment for the development of thinking in primary students with psychical developmental delay are quite large. An inclusive environment will become a developmental environment if the teacher properly organizes the learning process in the lesson, with the focused work of a teacher-psychologist, with the help of parents. Focusing on a well-performing normally developing peer, children with psychical developmental delay have every opportunity to rise to a higher level of development and significantly improve their mental abilities. The manifestation of greater activity in the learning process in an inclusive class provides a more successful schooling, as well as the prevention of secondary deviations in the development of this category of children.

\section{References}

1. T.A. Vlasova, V.I. Lubovsky, N.A. Tsypina, Children with psychical developmental delay (Moscow, 1984)

2. T.V. Egorova, Features of memory and thinking of primary students lagging behind in development (Moscow, 1973)

3. V.V. Lebedinsky, Disorders of mental development in children (Moscow,2003).

4. B.P. Puzanov, N.P. Konyaeva, B.B. Gorski et al, Teaching children with intellectual disabilities: (oligophrenopedagogy) (Moscow, 2008)

5. N.V. Babkina, Almanac of the Institute of Correctional Pedagogy, 34, (2018)

6. E.F. Zambacevichene Defectology. 1, 28-33, (1984)

7. E.V. Zaika, N.P. Nazarova, I.A. Marenich, Psychology Issues. 1, 41-45, (1995)

8. A.Z. Zak Methods of development of intellectual abilities in children 9 years old (Moscow, 1994)

9. O.V. Solovieva, Cognitive abilities of students: phenomenology, diagnosis and development (Stavropol, 2010)

10. G.A. Uruntaeva, J.A. Afonkina, Workshop on child psychology (Moscow, 1995) 